\title{
Psicooncología
}

ISSN: $1696-7240$

\section{Uso de biofeedback de variabilidad de la frecuencia cardiaca durante la radioterapia como método de distracción cognitiva y autorregulación en un paciente pediátrico: Informe de caso}

\author{
Mariana Alejandra Sierra Murguía ${ }^{1, *}$; Amalia Padilla Rico²; Juan Manuel Fraga Sastrías ${ }^{3}$
}

Recibido: 20 de junio de 2017 / Aceptado: 25 de agosto de 2017

Resumen. Objetivo: Evaluar la efectividad del uso de biofeedback de variabilidad de frecuencia cardiaca (VFC) durante la radioterapia como método de distracción cognitiva y autoregulación emocional en un paciente pediátrico. Método: La paciente es una niña de 11 años con diagnóstico de sarcoma de Ewing en la pierna izquierda. Recibió 28 sesiones de radioterapia. Antes de iniciar tratamiento, se evaluó psicológicamente a la paciente con la entrevista Mini Kid, identificando que no tenía psicopatología. Dos semanas previas a iniciar, se evaluó la frecuencia cardiaca (FC) y variabilidad (VFC) de la misma (indicadores que han sido utilizados previamente como correlatos fisiológicos de estrés y relajación) con un software especializado llamado EmWave, identificando que sí presentaba activación fisiológica asociada a estrés. Se hizo entrenamiento en respiración diafragmática apoyado con el equipo de retroalimentación dos semanas previas a la radioterapia. El día que inició tratamiento, se volvió a evaluar la frecuencia y variabilidad antes de comenzar. Durante la radioterapia se instrumentó a la paciente al software y se permitió que ella escogiera el juego (que registra las variables de FC y VFC e interactúa conforme ella logra autoregulación fisiológica) y éste era proyectado en el techo de la sala mientras recibía la radiación. Resultados: Se identificaron cambios en la variabilidad de la frecuencia cardiaca, logrando una autoregulación, mostrando así que la paciente aprendió a relajarse y que lo puso en práctica durante la radioterapia. Conclusión: El uso del biofeedback es innovador en el tratamiento con radioterapia; permite al paciente integrar los dos métodos más utilizados como preparación para la misma: distraer el foco atencional y lograr una autoregulación. Se sugiere ampliar la muestra para conocer los efectos y generalizar los resultados.

Palabras clave: Radioterapia; preparación psicológica; retroalimentación biológica; autoregulación; variabilidad de la frecuencia cardiaca.

\section{[en] Biofeedback use of heart rate variability during radiotherapy as a method of cognitive distraction and self-regulation in a pediatric patient: A case report}

Abstract. Aim: To assess the effectiveness of heart rate variability (HRV) biofeedback during radiotherapy as an intervention to promote cognitive distraction and emotional regulation un a paediatric patient. Method: Patient is an 11 years old female with Ewing Sarcoma on left leg. She recieved 28 radiotherapy

\footnotetext{
Cancer Center Tec100. Santiago de Querétaro. México

2 Cancer Center Tec 100. Santiago de Querétaro. México

E-mail: apadilla@cancercentertec100.com

3 Director General Cancer Center Tec100.

E-mail:jmfraga@cancercentertec100.com

* Dirección de correspondencia: Mariana Alejandra Sierra Murguía. Cancer Center Tec100: Hospital Médica Tec100, Prol. Priv. Ignacio Zaragoza No 16, Col Centro, Querétaro, Qro. México CP 76000. E-mail: msierra@ cancercentertec100.com
} 
sessions. Before initiating treatment, the patient was assessed with Mini International Neuropsychiatric Interview KID (MINI KID). She did not have a psychiatirc diagnostic, nor significative anxiety as rated by these instruments. Two weeks prior initiating radiotherapy, Heart rate (HR) and heart rate variability (HRV) (measures that have been used previously as physiologic corralates of stress and relaxation) were measured with a specialized software called EmWave. Results identified that there was a psysiologic activation related to stress. At the same session, the patient was trained in diaphragmatic breathing assisted with HRV biofeedback. The day the radiotherapy treatment started, HR and HRV were assessed an hour before. During radiotherapy, patient was instrumented to the software and the display was projected on the ceiling so that she could interact with the program at the same time she recieved radiotherapy. Patient was asked to choose the display she prefered (presented as interactive games by EmWave) and HR as well as HRV were measured during treatment. Results: Changes in HRV during radiotherapy were presented, these changes have been reported as indicative of self regulation, reflecting that the patient learned this behavior and was able to implement it during her treatment. Conclussion: The use of biofeedback is innovative in treatment with radiotherapy; it allows the patient to integrate the two most used methods for psychological preparation: attentional distraction and self regulation methods. It's suggested to amplify the sample in order to learn more about the use of biofeedback in radiotherapy and generalize results.

Keywords: Radiotherapy; psychological preparation; biofeedback; self regulation and heart rate variability.

Sumario. 1. Introducción 2. Método 3. Evaluación 4. Intervención 5. Resultados 6. Discusión 7. Conclusiones 8. Referencias bibliográficas.

Cómo citar: Sierra Murguía MA, Padilla Rico A, Fraga Sastrías JM. Uso de biofeedback de variabilidad de la frecuencia cardiaca durante la radioterapia como método de distracción cognitiva y autoregulación en un paciente pediátrico: reporte de caso. Psicooncología 2017;14:255-266. Doi: 10.5209/PSIC.57084.

\section{Introducción}

Se estima que en México, al año, existen aproximadamente 5.000 nuevos casos de cáncer en menores de 18 años y que la sobrevida estimada es del $56 \%{ }^{(1)}$. El tratamiento brindado para el cáncer infantil dependerá del tipo de cáncer y de la etapa clínica. Los tratamientos comunes son: quimioterapia, radioterapia, cirugía, inmunoterapia y trasplante de células madre. Los procedimientos médicos pediátricos se han asociado con altos niveles de ansiedad anticipatoria y distrés (malestar emocional). El manejo del malestar emocional relacionado a los procedimientos médicos, frecuentemente incluye el uso de técnicas de distracción atencional que se enfocan en cambiar el foco de atención del procedimiento hacia alguna actividad o tarea ${ }^{(2)}$ y el uso de técnicas de relajación, como respiración diafragmática e imaginación guiada ${ }^{(3)}$.

La radioterapia es una modalidad de tratamiento para el cáncer en niños, puede ir acompañado de cirugía y/o quimioterapia. La administración de la misma no es dolorosa, sin embargo, se acompaña de una serie de retos para el paciente pediátrico ${ }^{(4)}$. La radiación debe ser brindada de una manera precisa, de tal manera que se asegure que la dosis llegue al tumor, protegiendo en mayor medida posible el tejido sano. En población pediátrica esto es importante, ya que la radiación puede tener un impacto en el cuerpo que aún se encuentra en desarrollo y pueden quedar secuelas. Tomando esto en cuenta, se requiere que el paciente adopte la misma posición durante todo el tratamiento (sin moverse), y dependiendo de la 
zona a radiar, se pueden utilizar métodos de fijación (por ejemplo, una máscara termogénica). El equipo utilizado es grande y puede ser imponente para el paciente, quien además debe entrar solo al tratamiento, que puede generar ansiedad de separación ${ }^{(5)}$. Debido a esto, es frecuente que los niños desarrollen desagrado, ansiedad anticipatoria y resistencia al tratamiento ${ }^{(6)}$ y que, por lo mismo, en esta población se utilice sedación. Se ha reportado que la preparación psicológica para estos tratamientos es de suma importancia para reducir la ansiedad y promover cooperación al tratamiento ${ }^{(7)}$. Las intervenciones psicológicas para preparar a los pacientes a estos procedimientos, permiten al paciente aprender y practicar estrategias de afrontamiento que a su vez se relacionan con un incremento en la autoeficacia y disminución del malestar emocional ${ }^{(8)}$.

Existen tratamientos no farmacológicos que se han utilizado para disminuir el malestar emocional (distrés) y ansiedad que puede presentar esta población al recibir radioterapia. Algunas de estas estrategias incluyen brindar opciones en donde se involucre al niño en la toma de decisiones para incrementar la percepción de control (ej. Tipo de luz, película que le pondrán, colores, etc.) ${ }^{(9)}$, familiarizar a los pacientes con el equipo y lugar de tratamiento para disminuir el miedo a lo desconocido ${ }^{(6,7)}$ y utilizar distracción cognitiva para cambiar el foco de atención del procedimiento que genera estrés también es una estrategia efectiva para disminución de la ansiedad ${ }^{(10)}$. La función de la distracción, desde una perspectiva de condicionamiento clásico, reduce la respuesta de estrés (condicionado e incondicionado), cambiando la atención del estímulo incondicionado aversivo (ej. Radioterapia) y el estímulo condicionado previamente apareado con estrés (ej. Ârea de radioterapia, acelerador lineal, sala de espera, etc.). El contracondicionamiento busca introducir un estímulo que evoca conductas incompatibles con estrés (ej. Técnicas de relajación). Desde una perspectiva de condicionamiento operante, la distracción puede reforzar conductas de cooperación al procedimiento. Psicofisiologicamente, se ha descrito que la distracción modifica la percepción cognitiva de dolor alterando las respuestas nociceptivas ${ }^{(11)}$. Las técnicas de distracción pueden ser brindadas por diferentes medios (películas, conversaciones, juegos, etc.) que varían en el grado de actividad que debe hacer el paciente (activo/ pasivo). Se ha descrito que a mayor grado de actividad e interés que pueda generar, mayor potencial de distracción ${ }^{(12)}$ y por ende mayor control de la ansiedad. Es por esto que se decidió utilizar la retroalimentación biológica durante la radioterapia, pues involucra actividad e interés por parte del paciente, logrando distracción, además de promoviendo la autoregulación fisiológica.

La psicofisiología involucra el estudio científico de las interrelaciones entre procesos cognitivos y fisiológicos. Como psicofisiología aplicada, el biofeedback enseña a las personas a modificar respuestas fisiológicas mediante la retroalimentación de las mismas. Estas señales incluyen actividad muscular, flujo sanguíneo periférico, actividad cardiaca, electroencefalografía, presión sanguínea o actividad psicogalvánica de la piel ${ }^{(13)}$. Se define como la aplicación de métodos de condicionamiento operante al control de actividades viscerales, somatomotoras y del sistema nervioso central ${ }^{(14)}$. La premisa principal de la biofeedback es que permite al paciente, entrenado por medio de equipo especializado observar de manera amplificada, mediante sonidos o señales visuales en una pantalla cambios amplificados de sus propias respuestas fisiológicas que normalmente no son perceptibles y desarrollar la habilidad para autorregular las funciones corporales monitoreadas $^{(15)}$. 
El biofeedback ha sido utilizado en tratamientos con radioterapia para guiar la respiración en pacientes con cáncer de pulmón ${ }^{(16,17)}$, para disminuir rango de movimiento del tumor debido a la respiración en pacientes con cáncer de pulmón sometidos a resonancia magnética ${ }^{(18)}$, para disminuir el rango de movimiento en pacientes con radiocirugía estereotáctica para cáncer de hígado ${ }^{(19)}$.

El objetivo del presente trabajo fue evaluar la efectividad del uso de biofeedback de variabilidad de frecuencia cardiaca durante la radioterapia como método de distracción cognitiva y autoregulación emocional en un paciente pediátrico.

\section{Método}

Participante: Se trata de una niña de 11 años de edad con diagnóstico de Sarcoma de Ewing. El padecimiento inició en mayo 2016 con dolor en rodilla izquierda y limitación parcial a la deambulación, posteriormente notaron un tumor en muslo izquierdo en cara anterolateral. En junio del mismo año, se realizaron estudios de imagen y una biopsia por punción con reporte de Sarcoma de Ewing, se inició tratamiento con quimioterapia, completando 6 ciclos. En julio se hizo una resección quirúrgica del tumor y el reporte histopatológico refiere sarcoma de Ewing extra esquelético, sin evidencia de infiltración vascular intratumoral, bordes quirúrgicos libres de lesión. En diciembre del 2016, complementó quimioterapia, completando un ciclo más y en enero 2017 inició tratamiento con radioterapia, donde se prescribieron 28 sesiones.

\subsection{Hallazgos de la evaluación psicológica inicial}

Se realizó la evaluación antes de iniciar la radioterapia. Se aplicó la Entrevista MINI KID, que es una entrevista estructurada -que explora los principales trastornos del eje I del DSM IV ${ }^{(20)}$ y que ha sido validada para población mexicana por Heinze ${ }^{(21)}$ sin cumplir criterios diagnósticos para trastornos evaluados por el mismo. También se aplicó la Escala de Ansiedad Infantil de Spence ${ }^{(22)}$ en su versión para población mexicana, adaptada por Hernández-Guzmán et al., ${ }^{(23)}$ mostrando una puntuación total de 19 (sin ansiedad), con el puntaje más elevado para la subescala de ansiedad por separación (8), seguido de ansiedad generalizada (6), miedos (3), fobia social (2) $\mathrm{y}$ ataques de pánico y agorafobia, y trastorno obsesivo compulsivo (0).

\subsection{Definición de variables}

Ansiedad: Medida con escala de ansiedad infantil de Spence ${ }^{(22)}$, en su versión para población mexicana, adaptada por Hernández-Guzmán et al. ${ }^{(23)}$. Muestra una consistencia interna de Alfa de Cronbach de 0,88 y validez convergente de 0,70 (p $<0,0001)$.

Frecuencia Cardiaca: La frecuencia cardiaca se ha utilizado como correlato fisiológico de estrés en estudios previos ${ }^{(24)}$, en el presente estudio se utilizó una computadora portátil con el Software Emwave que utiliza un pletismógrafo que puede medir frecuencia cardiaca, conectado a un USB, esto permite la toma de datos de la variabilidad de la frecuencia cardiaca de la persona y facilita la información de manera gráfica e interactiva para el paciente. 
La variabilidad de la frecuencia cardiaca (VFC) se refiere a los cambios en el ritmo cardiaco que reflejan el control por el sistema cardiorrespiratorio. Es la variación en la frecuencia del latido o en la duración del intervalo $\mathrm{R}-\mathrm{R}^{(25)}$. Se ha descrito que estas fluctuaciones se sincronizan con la respiración y que a esto se le llama arritmia del sinus respiratorio y que éste refleja cambios en la regulación autonómica del corazón ${ }^{(26)}$. Un nivel óptimo de variabilidad de la frecuencia cardiaca refleja un funcionamiento saludable, capacidad de autoregulación y adaptabilidad; por el contrario, la poca variación puede ser indicador de depleción relacionada a la edad, estrés crónico, patología o funcionamiento inadecuado en varios niveles de control de la autoregulación, también se ha asociado a depresión y ansiedad ${ }^{(27)}$.

La estimación de la frecuencia cardiaca representa el efecto en red de la salida neural de los nervios parasimpáticos (vagos), que disminuyen la frecuencia cardiaca, y de los nervios simpáticos que lo aceleran. La frecuencia cardiaca, por ende, refleja el balance entre los sistemas simpático y parasimpático. Es importante destacar la relación entre frecuencia cardiaca y variabilidad de la misma, conforme se incrementa la FC, hay menos tiempo entre los latidos por lo que hay menos probabilidad de que se presente variabilidad; por el contrario, si disminuye la frecuencia cardiaca, hay mayor tiempo entre latidos y aumenta la variabilidad.

Variabilidad de la Frecuencia Cardiaca: Existe una relación entre la FC y la VFC, cuanto mayor es la FC, hay menos tiempo entre latidos (disminuyendo la variabilidad); por el contrario, si hay menor FC, hay mayor tiempo entre latidos (permitiendo mayor variabilidad). La interacción entre actividad autonómica neural, presión sanguínea, respiración y mayores niveles de sistemas de control producen cambios en ritmos tanto cortos como a largo plazo en medidas de $\mathrm{VFC}^{(28)}$. Las sociedades European Society of Cardiology y the North American Society of Pacing and Electrophysiology Task Force Report on HRV dividieron las oscilaciones en frecuencia cardiaca en 4 bandas de frecuencias: alta frecuencia (HF), baja frecuencia (LF), muy baja frecuencia (VLF) y frecuencia ultra baja (ULF) ${ }^{(29)}$.

- Banda de alta frecuencia (HF): frecuencia que varía de 0,15 a 0,4 Hz. Refleja actividad parasimpática y/o vagal, también se llama banda respiratoria, pues esta asociada a variaciones en la FC que se relaciona con la arritmia sinusal respiratoria, Una disminución en ondas de alta frecuencia (HF) se ha asociado con estrés, pánico, ansiedad y preocupaciones ${ }^{(30)}$.

- Banda de baja frecuencia (LF): El rango varía de 0,04 a 0,15 Hz, Esta región previamente era llamada rango baroreceptora o de frecuencia media, ya que refleja actividad baroreceptora ${ }^{(31)}$.

- Banda de muy baja frecuencia (VLF): rango entre 0,0033 y $0,04 \mathrm{~Hz}$. La baja presencia de ondas de muy baja frecuencia se ha asociado con altos niveles de inflamación, incremento en el riesgo de desenlaces adversos y mayor mortalidad en general. Se ha asociado a mayor salud y bienestar y a actividad parasimpática ${ }^{(27)}$.

\section{Evaluación}

La frecuencia y variabilidad de la frecuencia cardiaca fueron medidas en tiempo real utilizando el software EmWave realizando las siguientes evaluaciones: 
Evaluación 1. Se tomó una evaluación inicial basal dos semanas antes de iniciar el tratamiento con radioterapia. Éste consistió en 2 minutos con los ojos abiertos, 2 minutos con los ojos cerrados, 2 minutos hablando de un estresor emocional y por último 2 minutos emitiendo respuesta de relajación natural.

Evaluación 2. Se volvió a evaluar FC y VFC justo antes de iniciar RT. El día que la paciente iniciaba radioterapia, se citó una hora antes para hacer esta evaluación. En ésta se tomó FC y VFC y posteriormente se realizaron ejercicios de respiración diafragmática aprendidos previamente.

Ver el diagrama de la evaluación e intervención en la figura 1.

\section{Intervención}

Fase 1: Entrenamiento previo:

Dos semanas antes del tratamiento con radioterapia, después de la primera evaluación, se realizó un entrenamiento en respiración diafragmática para lograr que la paciente aprendiera a regular la respuesta fisiológica de estrés. Se explicó el programa EmWave y lo que evalúa, esto con el objetivo de que ella conociera e interactuara con el mismo.

Al hacer el entrenamiento en respiración diafragmática se instrumentaba a la paciente con el pletismógrafo, que se sincronizaba con la pantalla del equipo, y mostraba en tiempo real la frecuencia cardiaca y la variabilidad de la misma. De esta manera ella podía identificar el efecto de su respiración en las variables fisiológicas (FC y VFC), logrando así generar una respuesta de relajación mediante la respiración.

La paciente entonces recibió entrenamiento en respiración diafragmática, asistido con biofeedback dos semanas antes de iniciar el tratamiento con radioterapia. Esto, con el objetivo de hacer lo mismo durante la radioterapia.

\section{Fase 2: Durante Radioterapia}

Durante el tratamiento con radioterapia, se mostró la pantalla del programa de biofeedback en el techo de la sala, con un proyector, y se le colocó a la paciente el pletismógrafo que iba conectado al software, quién se encontraba acostada boca arriba en la camilla del acelerador lineal, de manera que durante el tratamiento pudiera ver los datos en tiempo real (Ver foto 1 ).

Antes de iniciar la sesión, se le pedía a la paciente que escogiera la pantalla del programa, que cuenta con diferentes juegos en donde interactúa según la FC y VFC. El programa EmWave tiene diferentes pantallas que puede seleccionar la paciente y que interactúan dependiendo de la FC y VFC, son presentadas como juegos que en tiempo real se mueven si la paciente logra una respuesta de relajación. Esto genera un reforzamiento contiguo y contingente en tiempo real de la respuesta, haciendo más probable que vuelva a ocurrir la misma.

El programa corría mientras ella estaba en sesión de radioterapia (que duraba aproximadamente 20 minutos) y registraba la FC y VFC durante este tiempo. Esto se hizo todas las sesiones de radioterapia (25) y se tomaron para analizar las sesiones 1, 13 y 25 .

Evaluación final: Se tomaron las medidas de FC y VFC de la última sesión de radioterapia y se volvió a aplicar el Inventario de Ansiedad Infantil de Spence. 


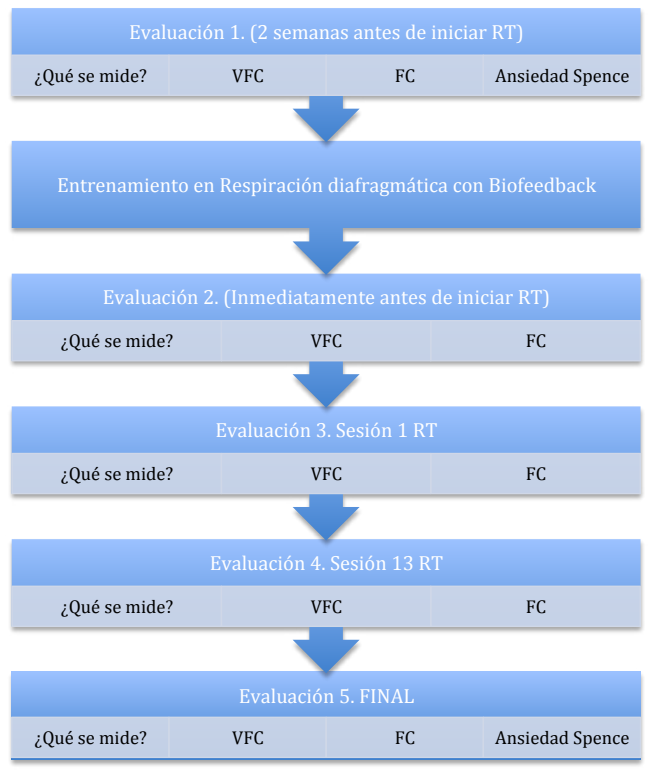

Figura 1. Diagrama de evaluación e intervención.

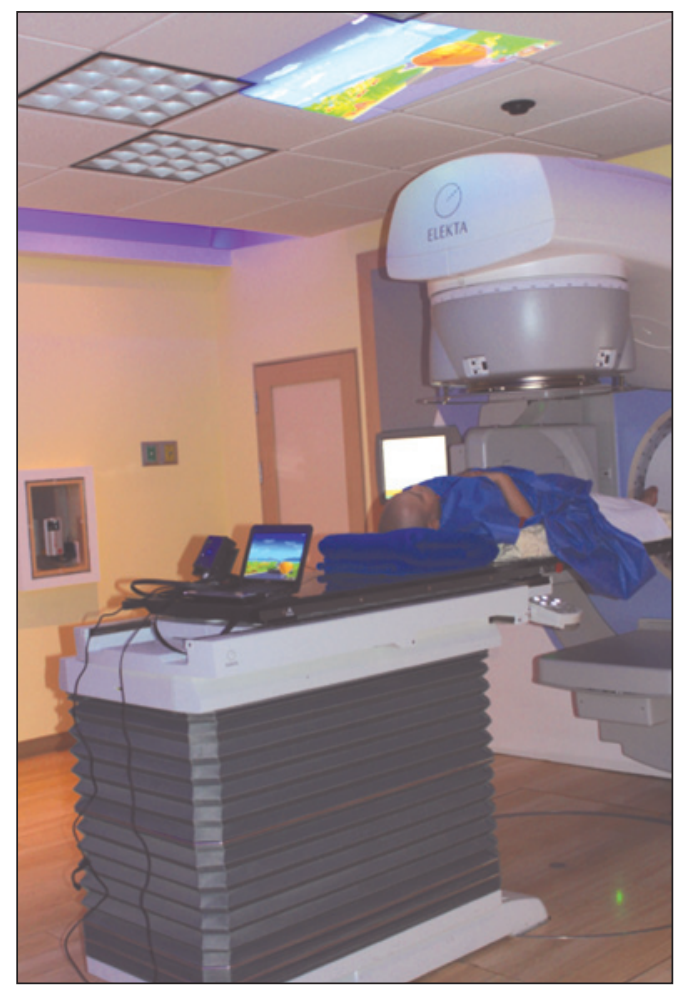

Foto 1. Posición de la paciente durante el tratamiento y colocación del equipo de retroalimentación biológica durante el mismo. 


\section{Resultados}

Durante la evaluación inicial, se determinó un puntaje de 19 en la escala de Ansiedad Infantil de Spence, predominando la ansiedad por separación con un puntaje de 8. En la segunda evaluación de la misma

En cuanto a los valores medidos con el software EmWave, se identificó una frecuencia cardiaca inicial de 104 y $100 \%$ de predominio de ondas de alta frecuencia (HF), predominando una actividad simpática antes de iniciar el tratamiento. Los resultados de la evaluación de la FC y VFC a lo largo del tratamiento se muestra en la tabla 1. En cuanto a VFC inició con $100 \%$ de ondas de alta frecuencia, mostrando un predominio en la actividad simpática. En la última sesión, obtuvo $100 \%$ de ondas de muy baja frecuencia (actividad parasimpática), mostrando un aprendizaje a lo largo de las sesiones (ver la figura 2).

Tabla 1. Descripción de Frecuencia Cardiaca y variabilidad de la misma a lo largo del tratamiento.

\begin{tabular}{lccccc}
\hline & $\begin{array}{c}\text { Evaluación } \\
1 \text { (Pre } \\
\text { tratamiento) }\end{array}$ & $\begin{array}{c}\text { Evaluación 2 } \\
\text { (Justo antes } \\
\text { de iniciar } \\
\text { tratamiento } \\
\text { con RT) }\end{array}$ & $\begin{array}{c}\text { Durante } \\
\text { sesión 1 }\end{array}$ & $\begin{array}{c}\text { Durante } \\
\text { sesión } \\
13\end{array}$ & $\begin{array}{c}\text { Durante } \\
\text { sesión } \\
25 \\
\text { (última } \\
\text { sesión) }\end{array}$ \\
\hline Frecuencia Cardiaca (lpm) & 104 & 98 & 92 & 98 & 100 \\
\hline $\begin{array}{l}\text { Banda de muy baja } \\
\text { frecuencia (VLF) }(\%)\end{array}$ & 0 & 7 & 88 & 86 & 100 \\
\hline $\begin{array}{l}\text { Banda de baja frecuencia } \\
\text { (LF) }(\%)\end{array}$ & 0 & 14 & 11 & 14 & 0 \\
\hline $\begin{array}{l}\text { Ondas de alta frecuencia } \\
\text { (HF) }(\%)\end{array}$ & 100 & 79 & 2 & 0 & 0 \\
\hline
\end{tabular}

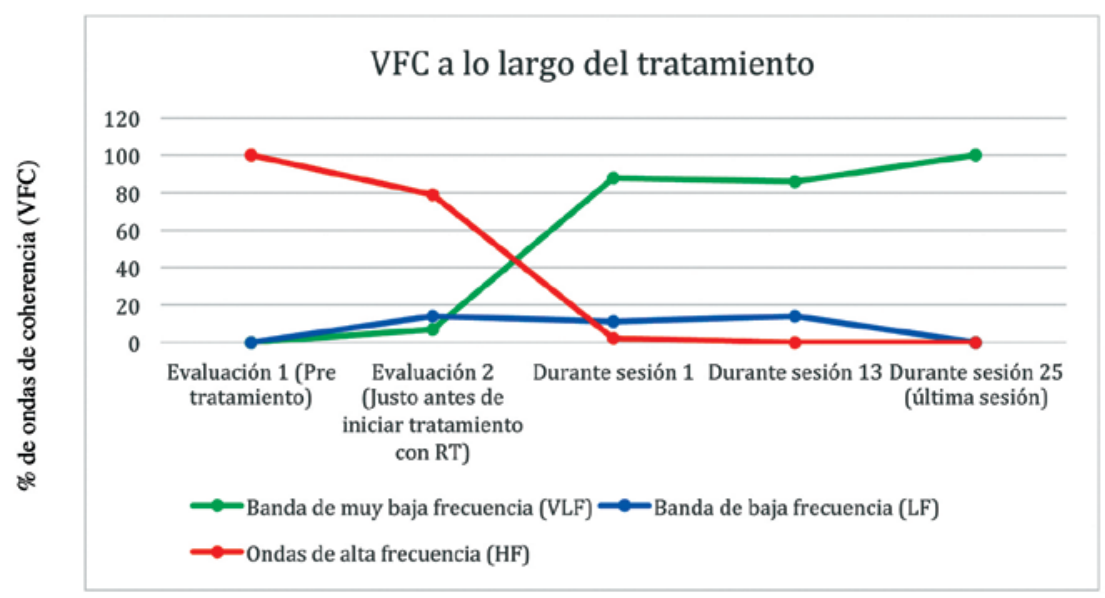

Figura 2. VFC a lo largo del tratamiento. 
Reporte subjetivo: Durante las sesiones de radioterapia, no se reportaron movimientos por parte de la paciente que requirieran detener el tratamiento. Presentó conducta de cooperación durante las 25 sesiones. La paciente refirió motivación por controlar la respuesta física de estrés y lograr dominar los juegos que se presentaban en las pantallas, refirió la entretenía durante el tratamiento con radioterapia ya que era visto como un reto.

\section{Discusión}

Para reducir el movimiento que generan los pacientes durante la radioterapia, así como en estudios como la resonancia magnética, se ha utilizado la sedación. Sin embargo, se ha descrito que el uso de terapias conductuales como técnicas de distracción y relajación pueden disminuir la necesidad de la misma ${ }^{(7)}$. Para esto, se sugiere la preparación psicológica para estos procedimientos e intervenciones, permitiendo al paciente aprender nuevas estrategias de afrontamiento y brindándole la oportunidad de llevarlas a cabo, disminuyendo el malestar emocional que puedan generar y aumentando la autoeficacia y la percepción de control. Esto a su vez se ha relacionado con mayor cooperación por parte de los niños a sus tratamientos ${ }^{(8)}$. A partir de estas premisas, se identificó que el uso del biofeedback permitía el aprendizaje de nuevas estrategias de autoregulación, interactuando durante la radioterapia.

Es una intervención novedosa, pues para procedimientos médicos en pacientes pediátricos, se han reportado el uso de estrategias de distracción atencional ${ }^{(10,32)}, \mathrm{y}$ estrategias de regulación emocional, como respiración diafragmática e imaginación guiada $^{(3)}$, así como con hipnosis; mientras que, el uso de la retroalimentación biológica de variabilidad de la frecuencia cardiaca nos permite realizar ambas, logrando que la paciente coopere al tratamiento, sin emitir movimientos físicos que requieran detener el tratamiento y sin presentar ansiedad. En la literatura se ha reportado solamente un artículo que habla sobre el uso de la retroalimentación biológica como preparación para el malestar emocional pre procedimientos médicos en pacientes con cáncer ${ }^{(33)}$, sin embargo, no se utiliza durante los mismos, como en el presente caso.

El presente es un informe de caso, que se realizó como piloto para conocer la factibilidad de dicha estrategia. Se sugiere hacer un estudio con grupos de control y hacer una intervención con mayor alcance para conocer si los resultados pueden ser generalizables.

\section{Conclusiones}

El objetivo del estudio fue evaluar la efectividad del uso del biofeedback durante el tratamiento con radioterapia como método de distracción atencional y de regulación emocional. La paciente no mostró niveles de ansiedad elevados en la escala de Ansiedad de Spence antes de iniciar el tratamiento, sin embargo, sí mostro un predominio en ondas de muy baja frecuencia (actividad simpática) que se relaciona a activación fisiológica por estrés. Se logró un cambio en las mismas, predominando desde la primera sesión (después del entrenamiento en respiración diafragmática) hasta la última, ondas de alta frecuencia (relacionadas a actividad parasimpática), 
mostrando así, que la paciente logró aprender estrategias de autoregulación y las pudo realizar durante el tratamiento con la radioterapia. Logrando así, una conducta de cooperación y evitando movimientos durante el tratamiento que requirieran pararlo.

El biofeedback puede ser utilizado durante la sesión de radioterapia y es útil para promover la autoregulación en los pacientes. Es necesaria la intervención desde antes de la sesión inicial para entrenar la respuesta de autoregulación y familiarizar al paciente con el software y el equipo de retroalimentación.

\section{Referencias bibliográficas}

1. CENSÍA. Programa de atención específico: Cáncer en la infancia y la adolescencia 2013- 2018. 2013. Programa Sectorial de Salud. México. [Acceso 14 de julio de 2017]. Disponible en: http://juntoscontraelcancer.mx/jcc/wp-content/uploads/2017/06/136PAE-SS-CanceresVarios-CancerdeInfanciayAdolescencia.pdf

2. Piira T, Hayes B, Goodenough B. Distraction methods in management of children's pain: an approach based on evidence or intuition. Suffering Child 2002; 1:1-10.

3. Rheingans JI. A systematic review of nonpharmalogical adjunctive therapies for symptom management in children with cancer. J Pediatr Oncol Nurs 2007; 24: 81-94. doi: $10.1177 / 1043454206298837$.

4. Constine, LS, Tarbell NJ, Kun LE, Halperin EC. Pediatric radiation oncology. 5th edn. Philadelphia Pa: Lippincott Williams and Wilkins, 2011. doi: 10.1097/00004424198605000-00016.

5. Hudson M, Tyc V, Srivastava DK, Gattuso J, Quargnenti A, Crom RN \& Hinds P. Multicomponent behavioral intervention to promote health protective behaviors in childhood cáncer survivors: The protect study. Med Pediatr Oncol 2002; 38): 2-11. doi: 10.1002/ mpo. 10071.

6. Filin A, Treisman S, Bortz A. Radiation therapy preparation by a multidisciplinary team for childhood cancer patients aged 3 1/2 -6 years. Pediatr Oncol Nurs 2009; 26: 80-5. doi: $10.1177 / 1043454208328766$.

7. Edwards A, Arthurs O. Paediatric MRI under sedation: Is it necessary? What is the evidence for the alternatives? Pediatr Radiol 2011; 41:1353-64. doi: 10.1007/s00247011-2147-7.

8. Flowers S, Birnie K. Procedural Preparation and support as a standard of care in pediatric oncology. Peadiatric Blood Cancer 2015; 62: S694-S723. doi: 10.1002/pbc.25813.

9. Scott L, Langton F, O’Donoghue J. Minimising the use of sedation/anaesthesia in Young children recieving radiotherapy through an effective play preparation program. Eur $\mathrm{J}$ Oncol Nurs 2002; 6:15-22. doi: 10.1054/ejon.2001.0162.

10. DuHamel KN, Redd WH, Vickberg SM. Behavioral interventions in the diagnosis, treatment and rehabilitation of children with cáncer. Acta Oncol 1999; 38:719-34. doi: 10.1080/028418699432879.

11. McGrath PJ, Finley GA. Virtual Reality distraction during pediatric medical procedures. Pediatric Pain Letter 2006;8: 6-10.

12. MacLaren JE, Cohen LL. A comparison of distraction strategies for venipuncture distress in children. J Pediatr Psychol 2005; 30:387-96. doi: 10.1093/jpepsy/jsi062.

13. Schwartz M, Andrasik F, editors. Biofeedback: A Practitioner's Guide. 3rd Ed. NY: Guilford, 2003. doi: 10.5298/1081-5937-44.4.09. 
14. Shapiro D, Kamiya J, Stovya J, Miller N. Biofeedback and self-control. Chicago: Aldine Publishing Company, 1972.

15. Holroyd KA, Penzlen DB. Self management of recurrent headache. Division of Mental Health. Geneva: OMS, 1993.

16. An S, Yeo I, Jung J, Suh H, Lee KJ, Choi J, et al. The effect of breathing biofeedback on breathing reproducibility and patient's dose in respiration-gated radiotherapy. Progress in Medical Physics. 2013; 24(3):135-9. doi: 10.14316/pmp.2013.24.3.135.

17. Pollock S, Tse R, Martin D, McLean L, Cho G, Hill R, et al. First clinical implementation of audiovisual biofeedback in liver cancer stereotactic body radiation therapy. J Med Imaging Radiat Oncol 2015; 59: 654-6. doi: 10.1111/1754-9485.12343.

18. Lee D, Greer P, Ludbrook J, Arm J, Hunter P, Pollock S, et al. Audiovisual biofeedback improves cine-magnetic resonance imaging measured lung tumor motion consistency. Int J Radiation Oncol Biol Phys 2016; 94: 628-36. doi: 10.1016/j.ijrobp.2015.11.017

19. Pollock S, O’Brien R, Makhija K, Hegi- Johnson F, Ludbrook J, Rezo A, et al. Audiovisual biofeedback breathing guidance for lung cáncer patients recieving radiotherapy: a multiinstitutional phase II randomised clinical trial. BMC Cancer 2015b; 15: 526. doi: 10.1186/ s12885-015-1483-7.

20. Sheehan DV, Lecrubier Y, Harnett-Sheehan K, et al. Reliability and Validity of the MINI International Neuropsychiatric Interview: According to the SCID-P. Eur Psychiatry 1997;12:232-41. doi: 10.1016/s0924-9338(97)8(31)97-x.

21. Heinze M. Mini International Neuropsychiatric Interview (M.I.N.I.): Spanish version (South and Central America) 5.0.0DSM-IV.México, D.F.: Instituto Nacional de Psiquiatría, 2000.

22. Spence SH, Barrett PM, Turner CM. Psychometric properties of the Spence Children's Anxiety Scale with young adolescents. I Anxiety Disord 2003; 17:605-25. doi: 10.1016/ s0887-6185(02)00236-0

23. Hernández-Guzmán L, Bermúdez-Ornelas G, Spence S, González Montesinos MJ, Martínez-Guerrero J, Aguilar Villalobos J, et al. Versión en español de la Escala de Ansiedad para Niños de Spence (SCAS). Rev Latinoam Psicol 2010; 42: 13-24.

24. Jay S, Elliott CH, Fitzgibbons I, Woody P, Siegel S. A comparative study of cognitive behavior therapy versus general anesthesia for painful medical procedures in children. Pain. 1995; 62:3-9. doi: 0.1016/0304-3959(94)00216-2

25. Bernston GG, Bigger JT, Eckberg DL, Grossman P, Kaufmann PG, Malik M, Nagaraja HN, Porges SW, Saul JP, Stone PH, Van der Molen MW. Heart rate variability: origins, methods, and interpretive caveats. Psychophysiol 1997; 34:623-48. doi: 10.1111/j.14698986.1997.tb02140.x

26. Billman GE. Heart rate variability a historical perspective. Front Physiol 2011; 2:86. doi: 10.3389/fphys.2011.00086

27. McCraty R. Schaffer F. Heart rate variability: New perspectives on physiological mechanisms, assessment of self-regulatory capacity, and health risk. Glob Adv Health Med 2015;4:46-61. doi: 10.7453/gahmj.2014.073

28. Tomasino D, Bradley RT. The coherent heart: heart- brain interactions, psychophysiological coherence, and the emergence of system-wide order. Boulder Creek, CA: Institute of Heartmath, 2009.

29. Task Force of the European Society of Cardiology and the North American Society of Pacing and Electrophysiology. Heart rate variability standards of measurement, physiological interpretation, and clinical use. Circulation 1996; 93:1043-65. doi: 10.1161/01.cir.93.5.1043 